ORIGINAL ARTICLE

\title{
Prevalence and risk factors for transmission of infection among children in household contact with adults having pulmonary tuberculosis
}

\author{
M Singh, M L Mynak, L Kumar, J L Mathew, S K Jindal
}

Arch Dis Child 2005;90:624-628. doi: 10.1136/adc.2003.044255

See end of article for authors' affiliations ....................

Correspondence to: Dr M Singh, Additional Professor, Department of Pediatrics, Advanced Pediatrics Centre, PGIMER, Chandigarh 160012 India; meenusingh4@ rediffmail.com, jolaz@vsnl. net

Accepted 5 August 2004

\begin{abstract}
Aims: To study the prevalence of tuberculosis infection among children in household contact with adults having pulmonary tuberculosis, and identify the possible risk factors.

Methods: Children under the age of 5 years who were in household contact with 200 consecutive adults with pulmonary tuberculosis underwent tuberculin skin testing. Transverse induration of greater than $10 \mathrm{~mm}$ was defined as positive tuberculin test suggestive of tubercular infection. Infected children underwent chest radiography and analysis of gastric lavage fluid or induced sputum for detection of acid fast bacilli.

Results: Tuberculin test was positive in 95 of 281 contacts (33.8\%), of which 65 were contacts of sputum positive patients, while 30 were contacts of sputum negative patients. Nine of these children were diagnosed as having tuberculosis based on clinical features and/or recovery of acid fast bacilli; seven were in contact with sputum positive adults. The important risk factors for transmission of infection were younger age, severe malnutrition, absence of BCG vaccination, contact with an adult who was sputum positive, and exposure to environmental tobacco smoke.

Conclusion: The prevalence of tuberculosis infection and clinical disease among children in household contact with adult patients is higher than in the general population, and risk is significantly increased by contact with sputum positive adults.
\end{abstract}

$\mathrm{T}$ uberculosis is one of the leading causes of mortality and morbidity across all age groups throughout the world, especially in developing countries. ${ }^{1}$ The infection is almost exclusively transmitted through air from patients with pulmonary disease. ${ }^{2}$ The risk of transmission is greatest if the index case is "sputum smear positive", and is directly proportional to the bacillary density in respiratory secretions. ${ }^{3}$ Therefore, proximity and persistence of contact are major determinants of the risk of transmission of infection, and those living within the same household are at higher risk than casual contacts. ${ }^{4}$ Among household contacts, those who are very young ${ }^{5}$ and those with absolute or relative immunodeficiency states are at increased risk of acquiring infection from the index case. ${ }^{6}$ Delay in the diagnosis and treatment of patients increases the risk of disease transmission to their contacts. ${ }^{7}$

For these reasons, children in contact with adult patients are at highest risk of getting infected, and hence contact screening is important for early detection of transmission of infection. ${ }^{8}$ This is of great value not only to detect infected individuals but for the community as a whole, because a child with tuberculosis represents recent and ongoing transmission of infection within the community. Although infected children are usually sputum smear negative and hence do not contribute to the immediate spread of the disease, ${ }^{9}$ they form a pool of infection from which a significant number of future adult cases arise.

The goal of tuberculosis control programmes is to eliminate the disease by breaking the chain of transmission, ${ }^{10}$ which can be effectively achieved by rapid identification and effective treatment of infectious cases. ${ }^{11}$ Once these cases are detected, it is imperative to detect infected persons in contact with them so that the chain of transmission can be broken. Therefore, in recent years contact tracing has started gaining importance and is now incorporated into the Revised
National Tuberculosis Control Programme of the Government of India. ${ }^{12}{ }^{13}$ This study has been undertaken with the aim of studying the prevalence of tuberculosis infection among children in household contact with adults having pulmonary tuberculosis and to identify the possible risk factors for transmission.

\section{METHODS}

This prospective, hospital based, descriptive study was conducted over a period of 18 months, on children who were contacts of 100 consecutive "sputum smear positive" and 100 consecutive "sputum smear negative" adults with pulmonary tuberculosis diagnosed at our institution. The study was initiated with the approval of the institutional ethics committee and written informed consent of participants was obtained prior to enrolment; written informed consent of either parent was taken for inclusion of children.

Children under the age of 5 years who were household contacts of newly diagnosed adults with pulmonary tuberculosis constituted the study population. Household contact was defined as a child living in the same house as the adult patient. Adults were diagnosed on the basis of suggestive symptoms and signs, confirmed with either presence of tuberculosis bacilli on Ziehl-Neelsen staining of sputum (hereafter referred to as sputum positive patients) or diagnostic chest radiograph in the absence of tuberculosis bacilli in sputum specimens (referred to as sputum negative patients). Children who had been previously treated for tubercular infection, those with concurrent conditions identified as risk factors for tuberculosis (HIV infection, haematological, or reticulo-endothelial system malignancies), and those who were previously or currently on immunosuppressive drugs including corticosteroids were excluded. 
Table 1 Demographic characteristics of adult patients with tuberculosis

\begin{tabular}{llllllll}
\hline & \multicolumn{3}{l}{ Sputum positive patients $(\mathbf{n}=\mathbf{1 0 0})$} & & \multicolumn{3}{l}{ Sputum negative patients $(\mathbf{n}=\mathbf{1 0 0})$} \\
\cline { 2 - 4 } Age $(\mathbf{y})$ & Male & Female & Total & & Male & Female & Total \\
\hline$<30$ & 23 & 22 & 45 & 22 & 19 & 41 \\
$31-40$ & 29 & 10 & 39 & 23 & 18 & 41 \\
$41-50$ & 10 & 0 & 10 & 8 & 2 & 10 \\
$>50$ & 5 & 1 & 6 & 6 & 2 & 8 \\
Total & 67 & 33 & 100 & 59 & 41 & 100 \\
\hline
\end{tabular}

Adults presenting with history of low grade fever, chronic cough, lymphadenopathy, and other symptoms suggestive of tuberculosis underwent sputum testing and postero-anterior chest radiograph. Sputum was collected on three consecutive mornings, homogenised, smears prepared on new glass slides, and stained by Ziehl-Neelsen technique. Chest radiographs were examined for features diagnostic of tuberculosis by an experienced radiologist who did not have access to results of sputum analysis.

Detailed history and clinical examination of children less than 5 years old who were in household contact with adult patients was performed by one of the researchers with postgraduate training in paediatrics. This included history of fever and/or cough of greater than two weeks duration, failure to gain weight, loss of appetite, decline in weight, and symptoms of extra-pulmonary tuberculosis such as lymphadenopathy. History of BCG vaccination was especially enquired after, and scars examined. A detailed history was also recorded regarding exposure to environmental tobacco smoke. Height and weight measurements were made by a single observer, and malnutrition classified according to the Indian Academy of Pediatrics (IAP) classification: ${ }^{14}$ grade 1 , weight $71-80 \%$ of expected; grade 2, weight $61-70 \%$ of expected; grade 3, weight $51-60 \%$ of expected; and grade 4 , weight less than $50 \%$ of expected. Grades 1 and 2 were categorised as mild malnutrition and grades 3 and 4 as severe malnutrition. Height was measured using a stadiometer (erect position for children older than 2 years and supine position for younger children); weight was recorded using a single precalibrated beam balance. Each of the children underwent tuberculin skin testing, performed by the intradermal injection of 1 Tuberculin Unit of Purified Protein Derivative PPD-RT23 with Tween 80 (Government BCG Laboratory, Guindy, India) into the volar surface of the left forearm using a 26 gauge needle and disposable syringe. This was read 72 hours later in good light with the forearm slightly flexed. Induration was measured by the pen method,,$^{15}$ and transverse induration of greater than $10 \mathrm{~mm}$ was defined as a positive tuberculin test suggestive of tuberculosis infection. A single technician trained in the

Table 2 Demographic characteristics of children in household contact with adult patients

\begin{tabular}{lll}
\hline & $\begin{array}{l}\text { Contacts of sputum } \\
\text { positive patients } \\
\text { (n= 140) }\end{array}$ & $\begin{array}{l}\text { Contacts of sputum } \\
\text { negative patients } \\
\text { (n= 141) }\end{array}$ \\
\hline Mean age (years) & 3.23 & 3.45 \\
Children $<2$ years of age & $33(23.6 \%)$ & $17(12.1 \%)$ \\
Sex ratio (M:F) & $73: 67$ & $78: 63$ \\
No malnutrition & $45(32.1 \%)$ & $51(36.2 \%)$ \\
Mild malnutrition* & $54(38.6 \%)$ & $56(39.7 \%)$ \\
Severe malnutrition** & $41(29.3 \%)$ & $34(24.1 \%)$ \\
BCG scar present & $98(70 \%)$ & $112(79.4 \%)$ \\
\hline *Defined as grades I and II of IAP classification. \\
**Defined as grades III and IV of IAP classification.
\end{tabular}

administration and interpretation of tuberculin test performed the procedure in all children.

All children underwent postero-anterior erect chest radiography which was reported by a single experienced radiologist (unaware of the results of tuberculin testing) and labelled as consistent or not consistent with tuberculosis. Children with a chest radiograph consistent with tuberculosis were admitted and underwent gastric lavage after an overnight fast of eight hours. This was done by aspirating the stomach contents, instilling $50 \mathrm{ml}$ distilled water through a nasogatric tube, and aspirating again. ${ }^{16}$ Both aspirates were mixed, subjected to concentration and homogenisation as for sputum, and stained by the Ziehl-Neelsen technique.

\section{Statistical analysis}

Analysis of parameters between contacts of sputum positive and sputum negative patients was done using Student's $t$ test for continuous variables and the $\chi^{2}$ test (with or without Yates's correction) for qualitative variables. Multivariate logistic regression analysis with positive tuberculin test as dependent outcome was also done.

\section{RESULTS}

Table 1 shows the demographic characteristics of adult patients in the study. None of the families had more than one index case. The mean duration of symptoms prior to presentation was 6.4 months (range 1-24 months), there being no significant difference between sputum positive and negative patients. Seventy of the 200 adult patients were smokers; there was no significant difference between sputum positive and negative patients in the prevalence $(38 \%$ and $32 \%$ respectively) or duration of smoking, mean (SD) being 13.8 (5.9) and 14.3 (5.1) years respectively.

Sputum positive patients had 140 children under 5 years in household contact, while sputum negative patients had 14l, the total number of contacts being 281. This group constituted the population for analysis. Table 2 shows the demographic characteristics of household contacts. There was no significant difference between contacts of sputum positive and sputum negative patients with respect to age, sex ratio, nutritional status, and presence of BCG scar. A total of 210 contacts had BCG scar, and of the remaining 71 without a scar, parents of 32 reported that they had been administered BCG vaccine. However, this could not be confirmed in the absence of written record of vaccination. A total of 96 contacts $(34.2 \%)$ had no malnutrition, 110 had mild malnutrition (39.1\%), and 75 had severe malnutrition (26.7\%). Among 75 children with severe malnutrition, BCG scar was present in $64(85.3 \%)$.

Tuberculin test was positive in 95 of 281 contacts (33.8\%), of which 65 were contacts of sputum positive patients, while 30 were contacts of sputum negative patients; the difference was statistically significant $(\mathrm{p}<0.01)$. The effect of contact with a sputum positive patient on the occurrence of positive tuberculin test was analysed and found to be highly significant $(\mathrm{p}<0.0001)$, with an odds ratio of 3.20 (95\% CI 1.84 to 5.60 ). Of children who tested tuberculin positive, $45 \%$, 
Table 3 Possible risk factors affecting prevalence of infection among children in household contact with adult patients

\begin{tabular}{lllll}
\hline Variable & Infection & No infection & p Value & Odds ratio (95\% Cl) \\
\hline Age $<2$ years $(n=50)$ & 35 & 15 & $<0.0001$ & $6.65(3.23$ to 13.79$)$ \\
Severe malnutrition $(n=75)$ & 43 & 32 & $<0.0001$ & $3.97(2.20$ to 7.21$)$ \\
Absence of BCG scar $(n=71)$ & 33 & 38 & 0.0136 & $2.07(1.14$ to 3.74$)$ \\
$\begin{array}{l}\text { Contact with sputum positive adult } \\
\text { ( } \mathrm{n}=140)\end{array}$ & 65 & 75 & $<0.0001$ & $3.20(1.84$ to 5.60$)$ \\
Exposure to smoking $(n=100)$ & 45 & 55 & 0.0003 & $2.68(1.52$ to 4.71) \\
\hline
\end{tabular}

$33 \%$, and $17 \%$ had induration of $10-15 \mathrm{~mm}, 15-20 \mathrm{~mm}$, and $>20 \mathrm{~mm}$ respectively.

Chest radiograph was consistent with tuberculosis in nine children, seven of whom were contacts of sputum positive patients. These children were diagnosed as cases of tuberculosis, and anti-tubercular therapy was initiated. Acid fast bacilli were not detected in any child.

The 95 infected children were contacts of 55 sputum smear positive and 23 sputum smear negative adult patients. The mean duration of symptoms in the adults was 7.0 and 4.7 months respectively $(\mathrm{p}>0.05)$. Among these adult patients, there was history of smoking in $25(45.5 \%)$ with sputum positive tuberculosis and $8(34.8 \%)$ sputum negative patients.

Tuberculin skin test was positive in $26.3 \%, 25.4 \%$, and $57.3 \%$ contacts with no malnutrition, mild malnutrition, and severe malnutrition respectively (fig 1). Figure 2 shows the size of induration of positive tuberculin skin test among contacts with increasing grades of malnutrition $(p<0.0001)$. Table 3 shows the risk of transmission of infection with various factors.

\section{DISCUSSION}

Although great progress has been made in diagnostic methods for tuberculosis, screening tests to identify infection in early stages still rely on tuberculin sensitivity testing. One of its great limitations is that it depends on operator training and experience for correct administration and interpretation, which can significantly alter results. However, this was unlikely to be a problem in our study as a single trained technician with several years experience performed the test in all children; hence the test was not read by multiple independent observers. Thus, although operator bias cannot be definitely excluded, the study has shown that about one third of children in household contact with adult patients are infected with the organism and about 5\% are suffering from the disease. This is an important observation, particularly for a country where about $30 \%$ of the population under the age of 14 years in the general population shows a positive tuberculin test. ${ }^{17}$ It may be argued that the high prevalence of

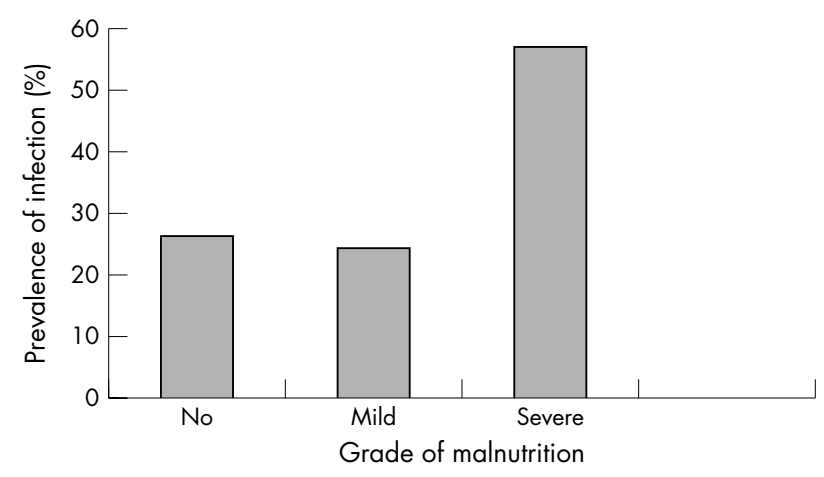

Figure 1 Prevalence of infection among various grades of malnutrition. positive tuberculin test in our study reflects this population characteristic rather than individuals at high risk of infection. Similarly, the effect of high coverage with BCG vaccine may also be a possible explanation. However, these theories would not be able to account for the significantly higher prevalence of positive tuberculin test among household contacts than in age matched counterparts with no contact. The effect of BCG is also not a plausible explanation as post-vaccination tuberculin test usually results in induration less than $10 \mathrm{~mm}$. Therefore, although the evidence is indirect, it is reasonable to conclude that tuberculin test positivity reflects infection in these children. This view is strengthened by the observation that twice the number of children in contact with sputum positive index cases had positive tuberculin test compared to those in contact with sputum negative cases. Likewise, the incidence of disease is almost four times higher in the former. Although it is generally believed that contacts of sputum positive patients have a higher prevalence of tuberculosis, there is no published information on transmission studied in a prospective fashion. In this study, the prevalence of infection among older children and adult contacts of index cases has not been calculated, but it is expected that a greater number of infected individuals will be detected by screening the entire family.

There is a greater proportion of relatively younger children who are contacts of sputum positive adults compared to sputum negative adults (33 $v$ 17). This is a valuable observation, which suggests that sputum positive adults probably transmit infection earlier than sputum negative persons. This is likely to have implications on the appropriate age for screening of childhood contacts; however, this observation needs to be confirmed in a larger study.

From our study, while it is not surprising that sputum positive index cases transmit infection, it is important to note that this occurs with sputum negative cases as well, although at a lower rate. This has also been observed by other workers previously, ${ }^{18}$ and abolishes the older concept that only "open

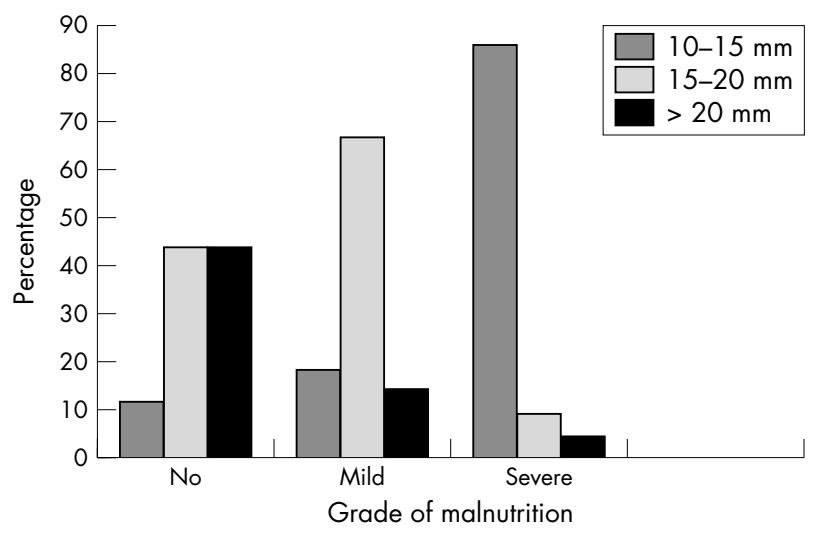

Figure 2 Size of induration of positive tuberculin test among various grades of malnutrition. 


\section{What is already known on this topic}

- Adult cases of pulmonary tuberculosis can transmit infection to family members in contact with them

- Transmission is believed to occur from sputum smear positive cases

- Malnourished and unvaccinated children are at higher risk of acquiring infection

cases" transmit infection. This observation has major implications on strategies for screening individuals at risk of infection and treating those who are infected. Obviously, a strategy of screening only those in contact with sputum positive cases is likely to miss about one third of infected individuals. A more appropriate population screening method would be to include household contacts of all persons diagnosed as having pulmonary tuberculosis. Another important implication is that it will be important to determine the role of these children in further transmission of infection, since by virtue of the fact that children are usually sputum negative, they are believed not to contribute significantly to transmission of infection. This aspect needs to be looked at and is an avenue for further research. A possible explanation for the transmission of infection from sputum negative patients is that they may not be negative in the strict sense of the term. A sputum sample must contain over $10000 \mathrm{AFB}$ per $\mathrm{ml}$ to be detected on Ziehl-Nielsen staining, whereas culture of specimens has been able to detect the organism even when bacillary density is as low as 100 per ml.

We have identified several risk factors that contribute to the transmission of tuberculosis infection among household contacts. Some of these have been previously described, ${ }^{19}{ }^{20}$ and confirmed by our study. Malnutrition seems to play a dual role with respect to tuberculosis. On the one hand, infection itself can result in failure to thrive and a malnourished state. ${ }^{8}$ On the other hand, hypersensitivity to tuberculin testing may be depressed in malnourished children, interfering with accurate interpretation of the test and resulting in false negative reports. ${ }^{21}$ However, our observations are in line with some authors who have not found a significant difference in the prevalence of positive tuberculin test among malnourished compared to normally nourished children, ${ }^{22} 23$ suggesting that a large number of malnourished children are able to respond to the tuberculin test. Nevertheless, we observed that the size of induration varied inversely with the nutritional status. While several normally nourished and mildly malnourished children had induration greater than $20 \mathrm{~mm}$, only a minority of severely malnourished children had induration greater than $15 \mathrm{~mm}$. This confirms the hypothesis that severe malnutrition depresses the hypersensitivity response to tuberculin, although some authors have been unable to detect such a difference. Our findings also suggest that the cut-off of $10 \mathrm{~mm}$ induration is applicable even in severely malnourished children, in contrast to what was previously believed.

The higher incidence of transmission of infection with exposure to environmental tobacco smoke is an interesting observation; a possible explanation is that cigarette smoke impairs pulmonary defence mechanisms, making airways more susceptible to infection. Another possible explanation is that this actually reflects poorer health related habits and sociocultural behaviour that may independently increase risk of infection, including tuberculosis. However, in our study, contacts of sputum positive smokers had a higher infection rate than sputum positive non-smokers, suggesting that there may be a real link between the two factors. This was
What this study adds

- There is significant transmission of infection from sputum smear negative adult patients to children in household contact with them

- The risk of transmission is increased with exposure to environmental tobacco smoke

- Even severely malnourished children are able to respond to tuberculin test, although the magnitude of response is less than in normally nourished children

confirmed by multivariate logistic regression; sputum positivity had almost perfect positive correlation, whereas exposure to tobacco smoke and malnutrition had moderately positive correlation with a positive tuberculin test. This is another area where further studies are indicated.

\section{Conclusion}

Our findings suggest that there is a high prevalence of infection among children in household contact with adult cases of tuberculosis. The risk is higher for contacts of sputum positive patients, but is significant for contacts of sputum negative patients. The detection of infection in the latter group has important implications for population screening and treatment strategies. Severe malnutrition, younger age, and absence of BCG vaccination are significant risk factors for the transmission of infection. Exposure to environmental tobacco smoke independently increases the risk of acquiring infection. This study argues strongly in favour of routine screening of all children in contact with adult patients of pulmonary tuberculosis, and identifies the group of children at highest risk who should be screened on a priority basis if there are constraints of resources and time.

\section{Authors' affiliations}

M Singh, J L Mathew, M L Mynak, L Kumar, Department of Pediatrics, Postgraduate Institute of Medical Education and Research, Chandigarh, India

S K Jindal, Department of Pulmonary Medicine, Postgraduate Institute of Medical Education and Research, Chandigarh, India

Competing interests: none declared

\section{REFERENCES}

1 Centers for Disease Control and Prevention. Estimates for future global tuberculosis and mortality. JAMA 1994:271:739-40.

2 Raviglione MC, Sinder DE, Kochi A. Global epidemiology of tuberculosis. Morbidity and mortality of a worldwide epidemic. JAMA 1995;273:220-5.

3 van Zwandenberg DF. The influence of the number of bacilli on the development of tuberculosis disease in children. Am Rev Respir Dis 1960;82:31-44.

4 Centers for Disease Control. A strategic plan for the elimination of tuberculosis. MMWR 1989;38(supp 13):1 s-25s.

5 Miller FJW, Seal RME, Taylor MD. Tuberculosis in children. Boston: Little Brown \& Co, 1963

6 Anon. Recommendation of the advisory committee for elimination of tuberculosis. Screening for tuberculosis and tuberculosis infection in high risk population. MMWR 1989:39(suppl R-8):1s-7s.

7 Glassroth J. The physician's role in tuberculosis prevention. Clin Chest Med 1989; 10:365-74

8 Altet MN, Alcaide J, Plans P. Passive smoking and the risk of pulmonary tuberculosis in children immediately following infection. A case control study. Tubercle Lung Dis 1996;77:537-44.

9 Avan R, Beyers N. Childhood tuberculosis in an urban population in South Africa; burden and risk factors. Arch Dis Child 1999;80:430-7.

10 Clancy L, Rieder HL, Enarson DA, et al. Tuberculosis elimination in the countries of Europe and other industrialized countries. Eur Respir J 1991; 4:1288-95.

11 Enarson DA. The international union against tuberculosis and lung disease model. National Tuberculosis Programmes. Tuber Lung Dis 1995;76:95-9. 
12 Khatri GR, Frieden TR. Controlling tuberculosis in India. N Engl J Med 2002;347:1420-5.

13 Central TB Division, Directorate General of Health Services. RNTCP status report. TB India, Ministry of Health and Family Welfare, New Delhi, 2003.

14 Nutrition Subcommittee of the Indian Academy of Pediatrics. Indian Pediatr 1972;9:360.

15 Jordan TJ, Sunderam G, Thomas L, et al. Tuberculin reaction size measured by the pen method compared to traditional palpation. Chest 1987;92:234-6.

16 Rattan A. Diagnosis II. Laboratory aspects. In: Seth V, ed. Essentials of tuberculosis in children. Delhi: Jaypee Brothers Medical Publishers, 1997:222-3.

17 Park K. Park's textbook of preventive and social medicine, 16th edn. Jabalpur: M/s Banarsidas Bhanot Publishers, 2000.
18 Behr MA, Warren SA, Salamon H, et al. Transmission of Mycobacterium tuberculosis from patients smear negative for acid fast bacilli. Lancet 1999;353:444-9

19 Lloyd AVC. Tuberculin test in children with malnutrition. BMU 1968:3:529-31.

20 Houk VN, Kent DC, Baker JH, et al. The Byrd Study. In depth analysis of micro outbreak of tuberculosis in a closed environment. Arch Environ Health 1968;16:4-6.

21 Chakraborty AK, Ganapathy KT, Rajalakshmi R. Effect of nutritional status on delayed hypersensitivity due to tuberculin test in children of an urban slum community. Ind J Tub 1980;27:115-19.

22 Chadha VK, Suryanarayana HV, Krishnamurthy NK, et al. Prevalence of undernutrition among periurban children and its influence on the estimation of annual risk of tuberculosis infection. Ind J Tub 1997:44:67-71.

23 Ganapathy KT, Chakraborty AK. Does malnutrition affect tuberculin hypersensitivity reaction in the community. Ind J Pediatr 1982;49:377-82.

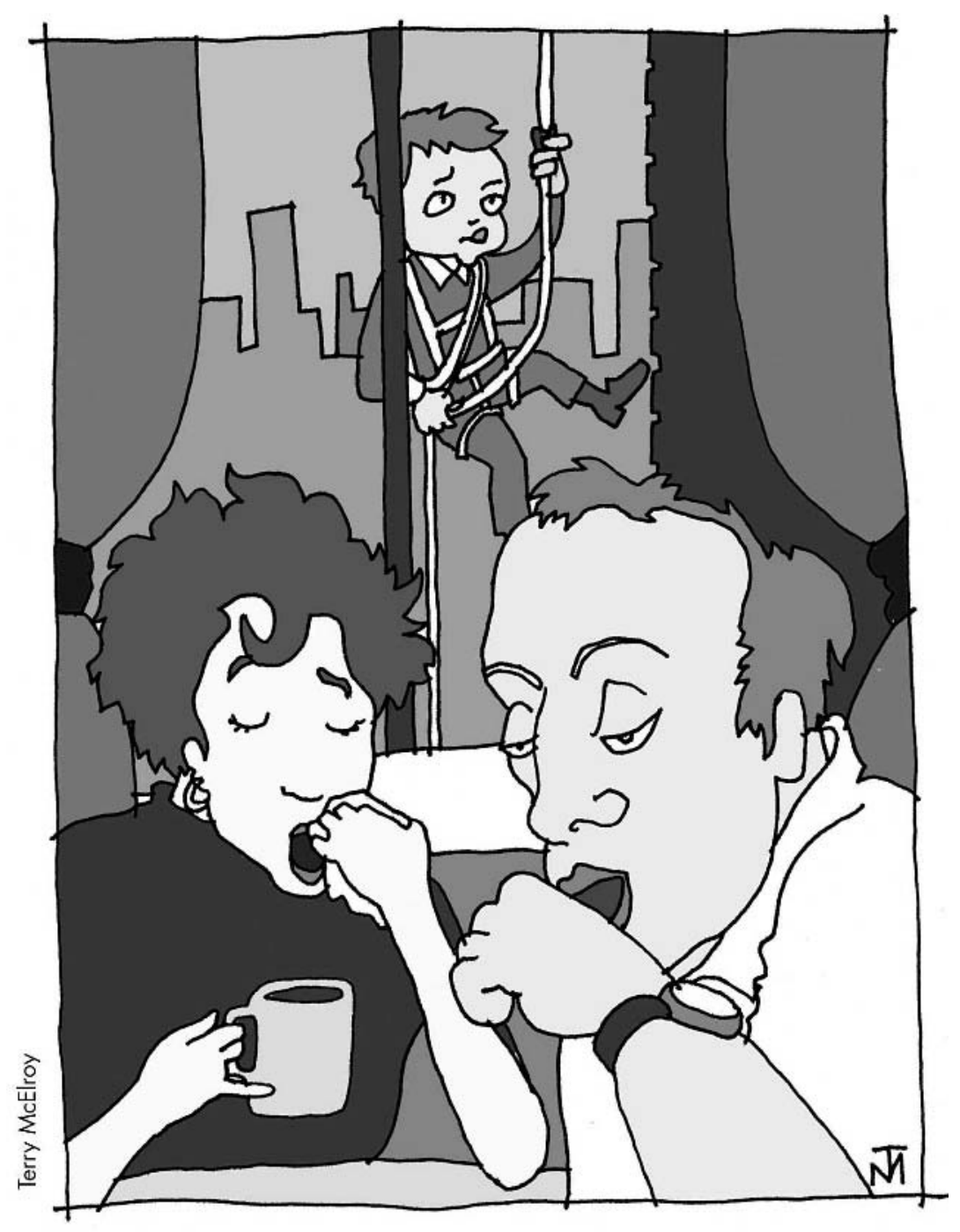

